\title{
Cannabis in fat: high hopes to treat obesity
}

\author{
Melody N. Hawkins ${ }^{1,2}$ and Tamas L. Horvath ${ }^{2,3}$ \\ 'Department of Internal Medicine, Section of Pulmonary, Critical Care, and Sleep Medicine, ${ }^{2}$ Yale University School of Medicine, and ${ }^{3}$ Department of Comparative Medicine, \\ Program of Integrative Cell Signaling and Neurobiology of Metabolism, New Haven, Connecticut, USA.
}

\begin{abstract}
Cannabinoid receptor type-1 (CB1) is known to have a substantial impact on the regulation of energy metabolism via central and peripheral mechanisms. In this issue of the $\mathrm{JCl}$, Ruiz de Azua and colleagues provide important insights into the regulation of adipocyte physiology by CB1. Mice with adipocyte-specific deletion of the CB1-encoding gene had an overall improved metabolic profile in addition to reduced body weight and total adiposity. These changes were associated with an increase in sympathetic tone of the adipose tissue and expansion of activated macrophages, both of which occurred prior to changes in body weight, lending support to a causal relationship between loss of CB1 in adipocytes and systemic metabolic changes. This work identifies adipocyte CB1s as a potential novel peripheral target for affecting systemic metabolism with diminished CNS effects.
\end{abstract}

\section{Effective obesity treatment} with tolerable side effects

Obesity is caused by a chronic imbalance between energy intake and expenditure. The growing incidence of obesity, especially in children and adolescents, is a major public health concern. Obesity amplifies the risk of developing metabolic syndrome, which has devastating consequences, including decreased quality of life and shortened life span. Additionally, obese individuals have increased rates of depression $(1,2)$. The pharmacologic treatment of obesity has been fraught with complications, including the inability to maintain weight loss, serious cardiovascular risks, and gastrointestinal symptoms (1).

Several pharmacologic therapies have been attempted to address the growing obesity epidemic. Unfortunately, the majority of these therapies have been associated with side effects that many would deem intolerable. Drugs that target pathways in metabolic tissues, such as adipocytes, liver, and skeletal muscle, show promise, but none have been clinically developed to date. Cannabinoid receptor type-1 (CB1) is a rational target for obesity treatment, giv- en its involvement in feeding control and peripheral metabolic regulation (3).

\section{The endocannabinoid system regulates energy metabolism}

The endocannabinoid system (ECS) is a neuromodulatory system composed of endogenously produced lipid cannabinoids that activate two well-characterized cannabinoid receptors, namely, CB1 and $\mathrm{CB} 2$, and several regulating enzymes (4). These cannabinoid receptors were first identified in 1988, and the first endogenous ligands, anandamide (AEA) and 2-arachidonoylglycerol (2-AG), were discovered in 1992 and 1995, respectively. The G protein-coupled CB1 is the most studied and relevant cannabinoid target (5) and is also the most abundant GPCR in the brain $(6,7)$. One function of $\mathrm{CB} 1$ is in the control of energy homeostasis. Induction of CB1 signaling stimulates feeding by increasing appetite, whereas blocking CB1 signaling induces hypophagia by an appetite-suppressant effect (5).

The cannabinoid receptor blocker rimonabant was the first successful obesity drug in this class (8) and was introduced in

Related Article: p. 4148

Conflict of interest: The authors have declared that no conflict of interest exists

Reference information: / Clin Invest. 2017;127(11):3918-3920. https://doi.org/10.1172/JCI97042.

Europe in June 2006 as Acomplia, promising to decrease appetite and body weight. Rimonabant showed effectiveness in the treatment of obesity and improved cardiometabolic risk factors $(2,8)$. Unfortunately, it was also associated with psychiatric side effects $(4,5)$ that were predicted based on a known mechanism of action. As a high-binding affinity CB1 antagonist with full inverse agonist activity, rimonabant blocked reward pathways and contributed to mood disorders and depression (4). In October of 2008, use of rimonabant was suspended in European nations due to the serious psychiatric side effects, and the drug is no longer used clinically.

The ECS regulates appetite and food intake via the hypothalamus, while the mesolimbic system modulates the reward function of food $(4,6)$. This system also acts on peripheral tissues to control metabolic functions (6). CB1 is expressed abundantly in the CNS and found peripherally in hepatocytes, adipocytes, skeletal muscle, and the pancreas $(3,8,9)$. Endocannabinoids and CB1s are present in peripheral cells and tissues that control energy homeostasis $(2,6)$. For example, in white adipose tissue, CB1 signaling is coupled to stimulation of lipoprotein lipase activity, suggesting that CB1 modulators act directly on adipose tissue and contribute to fat accumulation independently of the amount of food ingested (9). The next step in pharmacologic development of CB1-targeting drugs for treating obesity was to design a cannabinoid receptor antagonist that could not cross the blood-brain barrier in order to avoid CNS effects. While CB1 antagonists restricted to the periphery have been developed in rats (4), such antagonists have not been evaluated in humans.

In this issue of the JCI, Ruiz de Azua and colleagues provide valuable insight into CB1-dependent regulation of adipocyte physiology. Specifically, Ruiz de Azua and colleagues developed a mouse model in which adipocyte-specific deletion of the CB1-encoding gene could be induced. Using these mice, the authors 


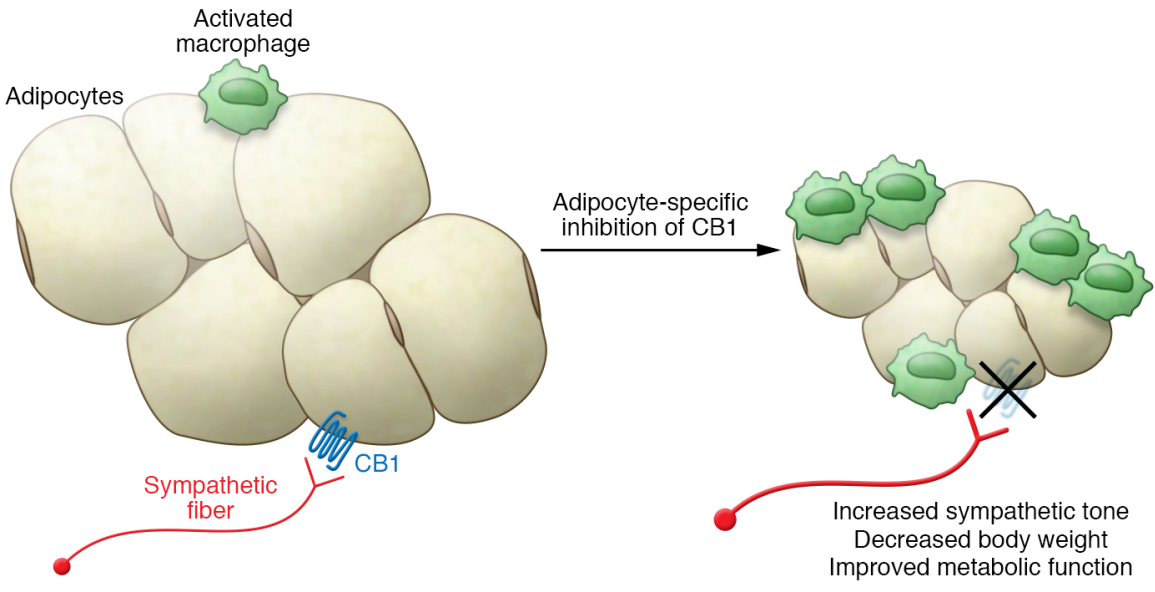

demonstrated that peripheral blockage of CB1 in adult obese mice caused fat redistribution, with lower total adiposity and weight loss. Additionally, loss of CB1 in adipocytes prevented diet-induced obesity and led to overall improved metabolic profile and browning of white adipocytes (10). These beneficial effects were accompanied by an increase in sympathetic tone of the adipose tissue along with expansion of activated macrophages, both of which occurred prior to changes in body weight (10). Together, these results lend support to and strengthen current evidence that suggests a causal relationship between loss of CB1 in adipocytes and systemic metabolic changes. Findings associated with CB1 blockade are summarized in Figure 1. A better understanding of this relationship provides much needed insight into the anticipated effects of peripherally restricted cannabinoid receptor antagonists on adipose tissue. Future work with similar experiments in hepatocytes, skeletal muscle, and the pancreas have the potential to better elucidate the effects of cannabinoid receptor antagonists in the periphery.

\section{Therapeutic implications: ECS system for body weight regulation}

Phytocannabinoids, namely $\Delta$ 9-tetrahydrocannabinol $(\triangle 9-\mathrm{THC})$ and the endocannabinoid AEA, are CB1 partial agonists that cause increased hunger sensation and stimulate food intake (11). Nabilone and dronabinol are synthetic $\triangle 9$-THC analogs that act as partial CB1 agonists and are FDA approved for the treatment of cancer and HIV cachexia for increasing appetite and body weight. Despite use in cases of severe
Figure 1. CB1 is essential for control of body weight and energy balance. Excessive CB1 signaling is associated with accumulation of adipose tissue, increased body mass, and hunger. In this issue, Ruiz de Azua and colleagues reveal that loss of CB1 specifically in adipocytes improves metabolic profile. These improvements are associated with an elevation of activated macrophages and increased sympathetic tone. This work supports further efforts in the development of peripherally restricted CB1 inverse agonists for treating obesity.

Melanin-concentrating hormone recepdisease states, these drugs continue to be used without significant side effects. Additionally, abuse of nabilione and dronabinol is an uncommon problem (11). The success of these synthetic cannabinoid receptor-targeting analogs proves the utility of pharmacotherapy in the management of appetite control. Despite the setbacks associated with negative psychiatric side effects after use of rimonabant, several alternatives, including CB1 partial agonists, neutral antagonists, allosteric modulators, and periphery-restricted CB1 antagonists (4), make cannabinoid-based therapy attractive for the management of obesity (3).

ECS overactivity is associated with metabolic and eating disorders that contribute to abdominal obesity, dyslipidemia, and hyperglycemia $(3,5,9)$. CB1 signaling inhibition decreases body weight in animal studies (11) by reducing food intake and abdominal adiposity. There is general interest in the idea of blocking the hyperactivity of the ECS as an obesity treatment (8).

Dronabinol has been approved for the treatment of HIV-induced wasting since 1992. In all studies, dronabinol mildly increased appetite; however, a marked improvement in mood was also noted. This observation raised the question of whether these effects are secondary to the appetiteregulating actions of cannabinoids or the result of a general improvement in the sense of well-being or a decrease in pain. Mounting evidence shows that ECS activation significantly increases body fat mass, despite minimal changes in food intake or appetite (6). The work of Ruiz de Azua et al. strengthens the hypothesis that the increased fat mass is due to a direct lipogenic action of CB1 activation (10). tor 1 (MCH-R1) antagonists have also shown promise as a pharmacotherapy for the treatment of obesity (1), as numerous studies suggest that melanin-concentrating hormone $(\mathrm{MCH})$ is involved in feeding and energy homeostasis (1). These neuropeptides are found in hypothalamic neurons, the peripheral nervous system, the immune system, intestine, and the reproductive system and are strongly expressed in the lateral hypothamalic area, a central area of feeding behavior regulation. Mice displayed hypophagia, increased metabolic activity, and weight loss after targeted deletion of $\mathrm{MCH}$. Overexpression of $\mathrm{MCH}$, on the other hand, led to increased body weight, hyperphagia, hyperlinsulinemia, and hyperglycemia after a high-fat diet (1). Rare genetic mutations that result in proopiomelanocortin (POMC) deficiency are characterized by hyperphagia and early onset lifelong obesity. In the hypothalamic leptin/melanocortin signaling pathway, melanocyte-stimulating hormone ( $\mathrm{MSH}$ ) transmits the anorexic effect of leptin through the melanocortin- 4 receptor. To date, there has been no effective treatment for the hyperphagia and early onset obesity; however, a recent brief report in the New England Journal of Medicine showed clinically significant weight loss in two female POMC-deficient patients following treatment with setmelanotide, a melanocortin-4 receptor agonist (12).

Unlike many classic neurotransmitters, endocannabinoids are not stored in synaptic vesicles (7). Instead, endocannabinoids are produced on demand from lipid precursors, often have a short halflife, and frequently work in a paracrine 
manner (5). Tight regulation of transcription and degrading enzymes also contributes to endocannabinoid production. Fatty acid amide hydrolase (FAAH) degrades AEA, while monoacylglycerol lipase (MAGL) is primarily responsible for the degradation of 2-AG. These enzymes are thought to link the ECS to a broader lipid signaling network. Further characterization of degrading enzyme regulation has the potential for identifying therapeutic targets outside the CB1 (5).

\section{Conclusions and future directions}

The primary defect associated with obesity is excessive endocannabinoid levels, not excessive CB1 expression (4); therefore, focusing on endocannabinoid levels rather than CB1 blockade has been proposed as a more physiological approach for obesity treatment $(4,8)$. This is especially so because there is concern that blockade of CB1 following treatment could adversely cause a feedback loop that would drive endocannabinoid levels even higher (4).

Other studies suggest that a polytherapeutic strategy may be the wave of the future (8). Additionally, several common diseases, such as hypertension, diabetes, and asthma, all use multiple medications to target different pathways in the treatment approach. As lifestyle, environment, and psychological factors possibly have a greater influence in obesity than in the aforementioned chronic conditions, a multitargeted treatment approach may be dictated for treating obesity. One reason the ECS system is an attractive therapeutic target is that this system modulates both food intake and energy expenditure (4) and current evidence implicates coordination between central and peripheral CB1s in the regulation of energy homeostasis (4).

Although an increased endocannabinoid tone has been associated with obesity $(4,5,9)$, it is also the assumption that drugs acting to increase endocannabinoid tone could be used to treat anxiety disorders and neurodegenerative diseases. The use of CB1 antagonists would need to be approached cautiously in patients with epilepsy, anxiety, or neurodegenerative disorders (6). However, the work by Ruiz de Azua et al. indicates that targeting CB1-mediated metabolic functions in adipose tissue remains an attractive obesity treatment option in order to avoid CNS side effects.

Address correspondence to: Melody Hawkins, 300 Cedar St., TAC S425 New Haven, Connecticut 06519, USA. Phone: 203. 824.4654; Email: melody.hawkins@yale. edu or melodynoleshawkins@gmail.com.

1. Cheon HG. Antiobesity effects of melanin-concentrating hormone receptor 1 (MCH-R1) antagonists. Handb Exp Pharmacol. 2012;(209):383-403.
2. Aronne LJ, Pagotto U, Foster GD, Davis SN. The endocannabinoid system as a target for obesity treatment. Clin Cornerstone. 2008;9(1):52-64.

3. Muller T, et al. Overactivation of the endocannabinoid system alters the antilipolytic action of insulin in mouse adipose tissue. Am J Physiol Endocrinol Metab. 2017;313(1):E26-E36.

4. Bermudez-Silva FJ, Viveros MP, McPartland JM Rodriguez de Fonseca F. The endocannabinoid system, eating behavior and energy homeostasis: the end or a new beginning? Pharmacol Biochem Behav. 2010;95(4):375-382.

5. Koch M. Cannabinoid receptor signaling in central regulation of feeding behavior: a minireview. Front Neurosci. 2017;11:293.

6. Pagotto U, Marsicano G, Cota D, Lutz B, Pasquali $\mathrm{R}$. The emerging role of the endocannabinoid system in endocrine regulation and energy balance. Endocr Rev. 2006;27(1):73-100.

7. Piomelli D. The molecular logic of endocannabinoid signalling. Nat Rev Neurosci. 2003;4(11):873-884.

8. André A, Gonthier MP. The endocannabinoid system: its roles in energy balance and potential as a target for obesity treatment. Int J Biochem Cell Biol. 2010;42(11):1788-1801.

9. Matias I, Di Marzo V. Endocannabinoids and the control of energy balance. Trends Endocrinol Metab. 2007;18(1):27-37.

10. Ruiz de Azua I, et al. Adipocyte cannabinoid receptor $\mathrm{CB} 1$ regulates energy homeostasis and alternatively activated macrophages. J Clin Invest. 2017;127(11):4148-4162.

11. Engeli S. Central and peripheral cannabinoid receptors as therapeutic targets in the control of food intake and body weight. Handb Exp Pharmacol. 2012;(209):357-381.

12. Kühnen $P$, et al. Proopiomelanocortin deficiency treated with a melanocortin-4 receptor agonist. N Engl JMed. 2016;375(3):240-246. 